\title{
Case Report: Ossified Subperiosteal Hematoma in the Humerus of a Lacrosse Player
}

\author{
Alana M Munger, MD*, Dieter M Lindskog, MD and Elizabeth C Gardner, MD \\ Department of Orthopaedics and Rehabilitation, Yale School of Medicine, USA
}

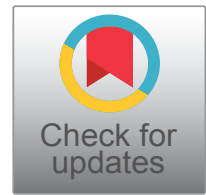

*Corresponding author: Alana M. Munger, MD, Yale School of Medicine, Department of Orthopaedics and Rehabilitation, 800 Howard Avenue, PO Box 208071, New Haven, CT 06520-8071, USA

\begin{abstract}
Case: An 18-year-old male collegiate lacrosse player presented to a sports medicine clinic with two-week history of atraumatic pain along the lateral aspect of his left arm. Radiographs demonstrated a thin, well-defined exostosis arising from the cortex of the mid-humeral diaphysis and magnetic resonance imaging demonstrated an osseous excrescence in the anterolateral aspect of the humerus and the prominence of the cortex in this area suggested a prior subperiosteal hematoma in this region - consistent with an ossified subperiosteal hematoma.
\end{abstract}

Conclusion: This case reports demonstrates the first description of an ossified subperiosteal hematoma within the mid-diaphyseal region of the humerus.

\section{Introduction}

Ossified subperiosteal hematomas represent a hemorrhagic collection between the cortex and periosteum of bone. Ossified subperiosteal hematomas have been described in the calvarium [1-4], tibia [5-8], femur $[5,9,10]$, and iliac bone [11-13]. Martinez-Lage and colleagues described a case of a 5-month-old boy who presented with sagittal synostosis due to a ossified cephalohematoma of the cranial vault who underwent bilateral parasagittal craniectomies and removal of the ossified hematoma en bloc [4]. Hui and colleagues described a case in which a 25 -year-old male sustained an inversion injury to his left ankle and subsequently developed a subperiosteal hematoma in the posterior distal tibia, which ossified with time [7]. The authors believed that the periosteum was detached from the distal tibia but remained intact during the sprain injury, which allowed for the subperiosteal hemorrhage to form [7].

An ossified subperiosteal hematoma of the humeral mid-diaphysis has not been described previously. As such, the purpose of this case report is to describe the clinical and radiographic features of an ossified subperiosteal hematoma of the humeral mid-diaphysis.

\section{Statement of Informed Consent}

The patient was informed that the data concerning this case would be submitted for publication and he provided consent.

\section{Case Report}

An 18-year-old male collegiate lacrosse player presented to a sports medicine clinic with two week history of pain along the lateral aspect of his left arm. He did not recall a specific episode of injury, although he explained the location of pain to be in the area of his arm exposed between his shoulder and elbow pads. He reported a two-year history of "calcification" within the muscle of his left arm, although it had not been painful previously. He associated the onset of this pain with his arrival at college and playing against bigger opponents.

On exam, there was an apparent firm and relatively mobile nodule within the lateral biceps musculature. The patient reported that this nodule was sore, but palpation and percussion of the lesion did not cause radiating pain or neurologic symptoms. There was no pain with palpation of the biceps tendon as it crossed the elbow or at the bicipital groove. The Speed's and

Citation: Munger AM, Lindskog DM, Gardner EC (2021) Case Report: Ossified Subperiosteal Hematoma in the Humerus of a Lacrosse Player. Int J Sports Exerc Med 7:208. doi.org/10.23937/24695718/1510208

Accepted: December 04, 2021; Published: December 06, 2021

Copyright: (C) 2021 Munger AM, et al. This is an open-access article distributed under the terms of the Creative Commons Attribution License, which permits unrestricted use, distribution, and reproduction in any medium, provided the original author and source are credited. 
a

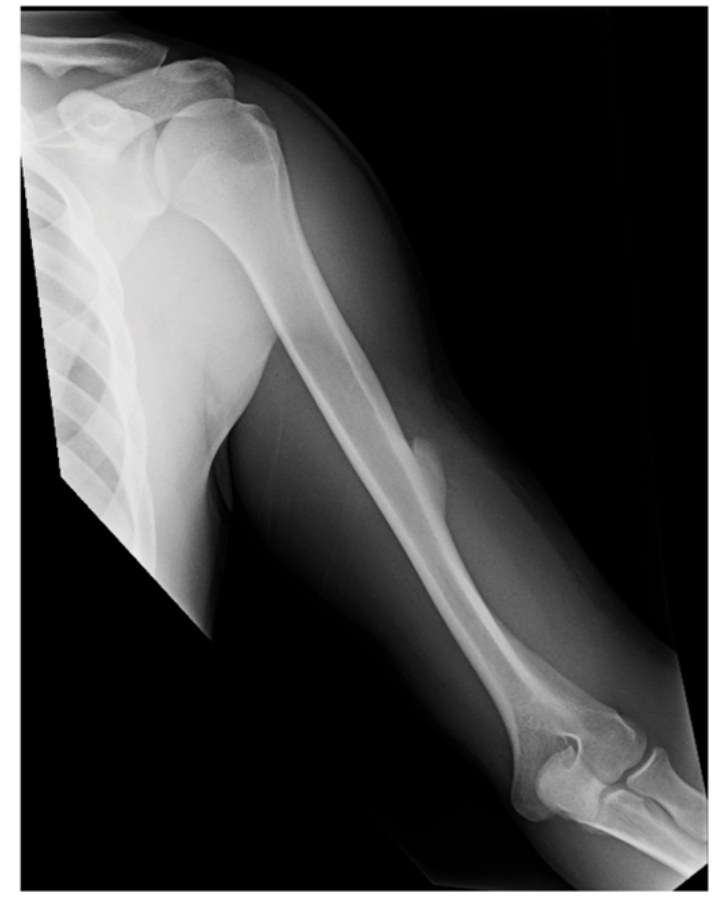

C

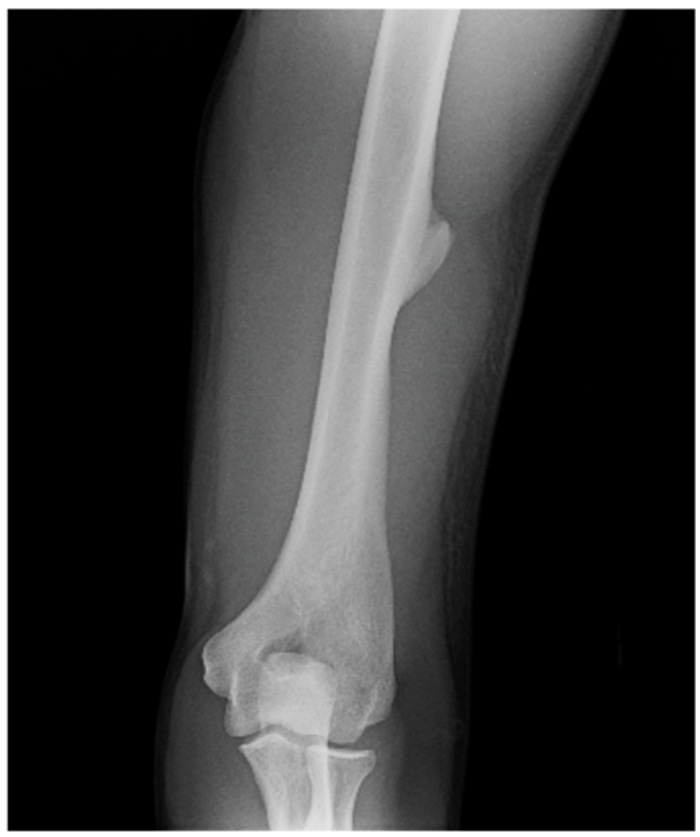

b

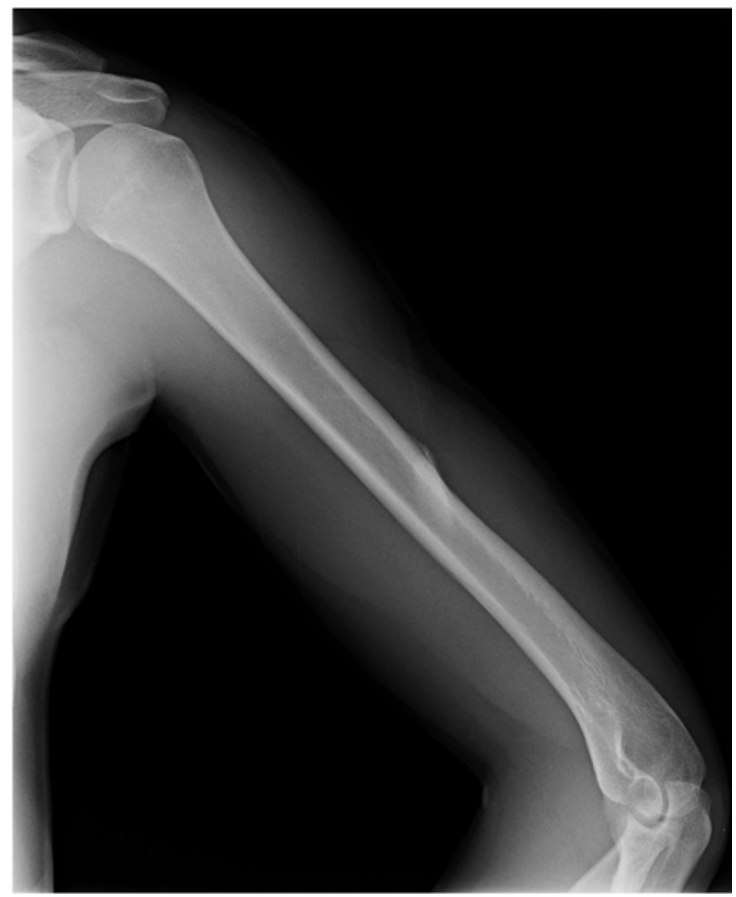

d

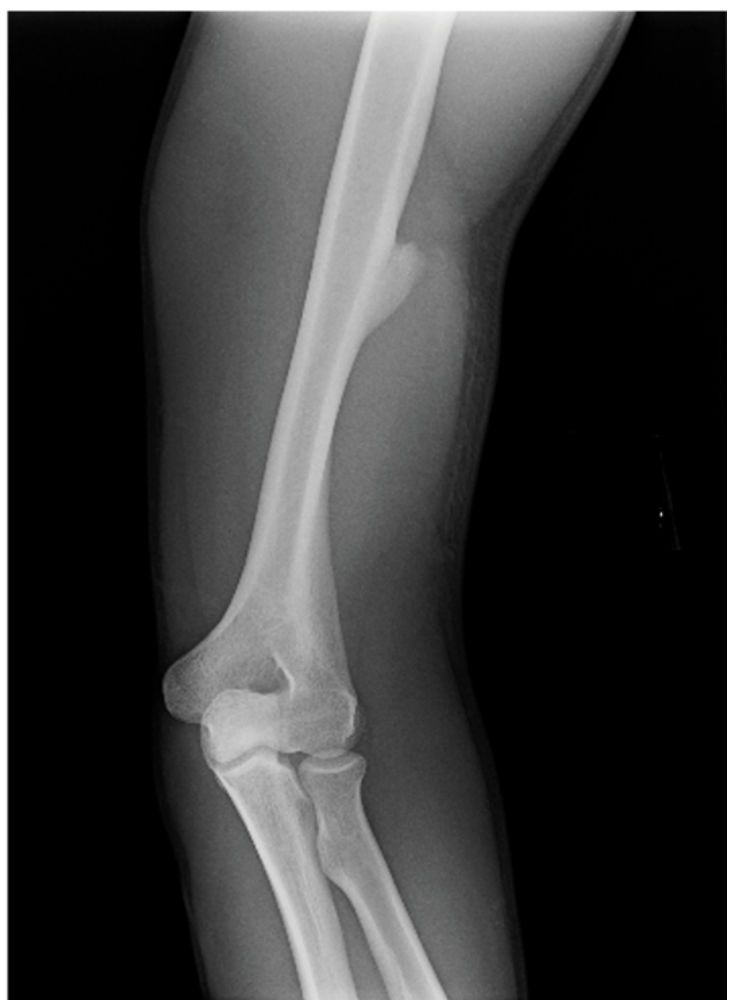

Figure 1: Antero-posterior (a), lateral (b), and orthogonal $(c, d)$ radiographs of the left humerus demonstrating a welldefined exostosis arising from the mid-humeral diaphysis at the insertion of the deltoid muscle. There is no evidence of fracture or other osseous abnormality.

Yeragson's tests were both negative. Pain was present with resisted elbow flexion, and the patient noted this pain to be in the area of the brachioradialis. The patient denied pain with pronation or supination of the forearm.

A seven-day course of oral anti-inflammatoryswas ordered and radiographs of the right arm were taken (Figure 1). Antero-posterior (a), lateral (b), and orthogonal $(c, d)$ radiographs of the left humerus demonstrated a well-defined exostosis arising from the mid-humeral diaphysis at the insertion of the deltoid muscle. After review of the radiographs, it was believed that the likely diagnosis was an ossified periosteal hematoma and it was recommended than Magnetic Resonance Imaging (MRI) of the left humerus with and without contrast be done to confirm the diagnosis. Given that there was no increase in risk of fracture, the patient was allowed to continue with unrestricted 

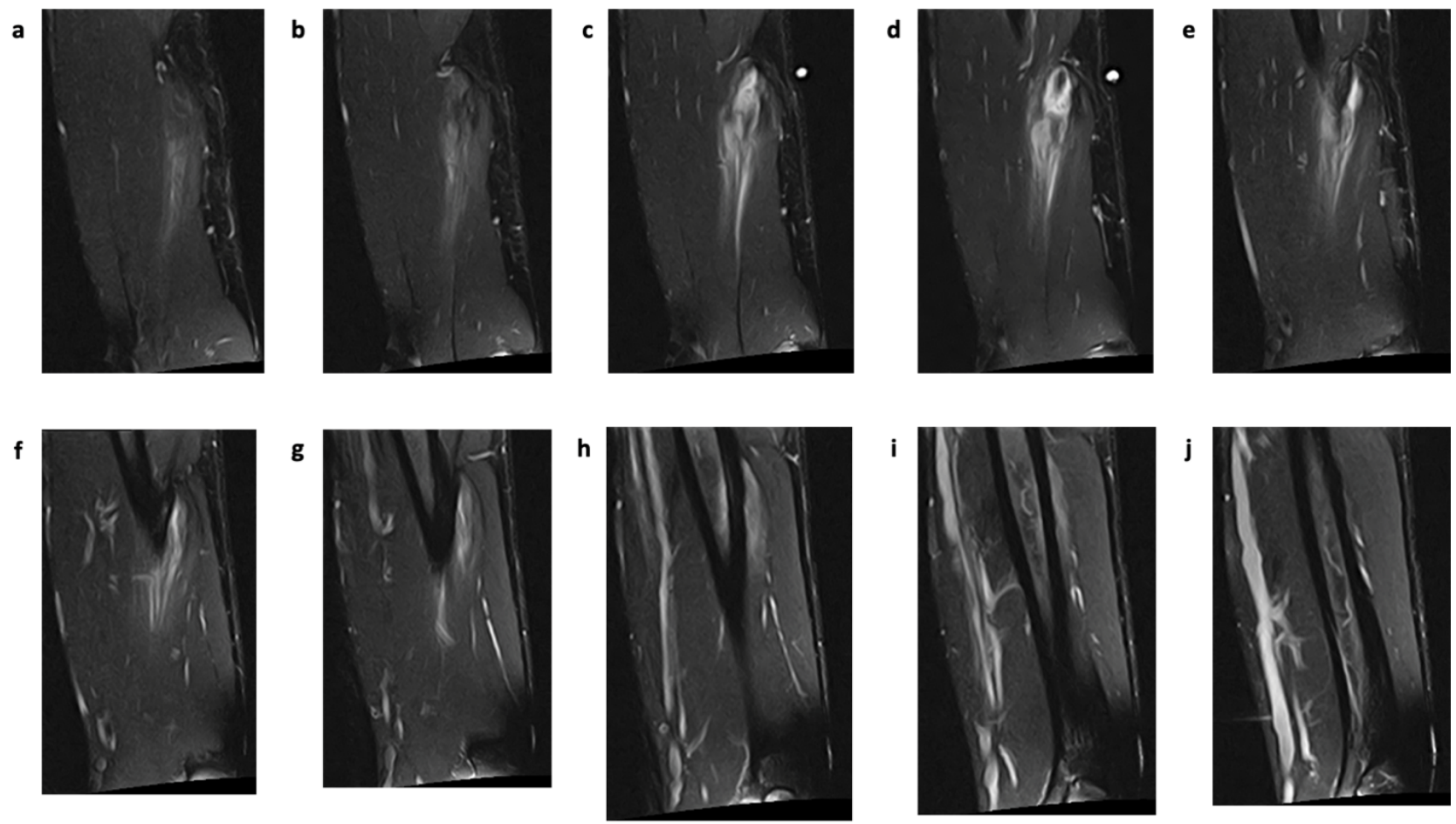

Figure 2: Coronal T2 FS MRI images (TR: 3,300 ms, TE: $48 \mathrm{~ms}$ ) from most anterior (a) to most posterior (j), revealing an osseous excrescence in the anterolateral aspect of the humerus near the deltoid attachment. FS: Fat Suppression.

participation in all lacrosse activities as tolerated. The area was padded for protection as needed.

Coronal (Figure 2) and axial (Figure 3) MR imaging of the left humerus demonstrated an osseous excrescence in the anterolateral aspect of the humerus near the deltoid attachment. The prominence of the cortex in this area suggested a prior subperiosteal hematoma in this region. The changes related to the tip of the osseous excrescence most likely represented an adventitial bursitis. With the diagnosis of an ossified periosteal hematoma confirmed, the patient was allowed to continue with unrestricted participation in all lacrosse activities as tolerated.

The patient was most recently evaluated for pain in the left acromioclavicular joint. At that time, twentynine months since his initial presentation for pain in the left humerus, the patient had been participating in lacrosse without any restrictions and only occasional local soreness. He had no pain with range of motion of the left elbow or wrist.

\section{Discussion}

The diagnosis of an ossified subperiosteal hematoma wasmade based on a combination of clinical and radiological features. The patient presented with atraumatic pain in the lateral aspect of his left arm and radiographs of the region demonstrated a well-defined exostosis arising from the mid-humeral diaphysis. Given the inability to definitively exclude myositis ossificans and parosteal osteosarcoma, a MRI of the left humerus was obtained, which demonstrated an osseous excrescence in the anterolateral aspect of the humerus near the deltoid attachment. The prominence of the humeral cortex in this area suggested a prior subperiosteal hematoma in this region, highly suggesting the patient's mass to be an ossified periosteal hematoma. This case demonstrates the first reported case of an ossified subperiosteal hematoma of the mid-diaphyseal region of the humerus bone.

Risk factors for the development of subperiosteal hematomas include:young age with a history of trauma, neurofibromatosis type 1 and hemophilia. For neurofibromatosis type 1 , the pathophysiology of the subperiosteal hemorrhage is thought to be secondary to a combination of vascular fragility and weakened periosteal attachment (due to mesodermal dysplasia, direct periosteal involvement by a plexiform neurofibroma, or a combination of the two) $[6,8]$. Hemophilic pseudotumors, defined as an encapsulated hematoma, can be secondary to intraosseous hemorrhage following direct trauma and are commonly seen in small cancellous bones such as the calcaneus, talus and metatarsals of the feet [14]. In the cases of patients without underlying conditions - as was the case with this patient - subperiosteal hematomas are thought to develop in children and young patients due to the loose attachment of the periosteum from the underlying bone [12]. A traumatic incident is thought to lead to a periosteal avulsion injury with damage to the nutrient vessels, resulting in subperiosteal hemorrhage [12].

The cause of ossification of the subperiosteal hematomas may in part be explained by the biology of fracture healing, which involves the coordinated action 

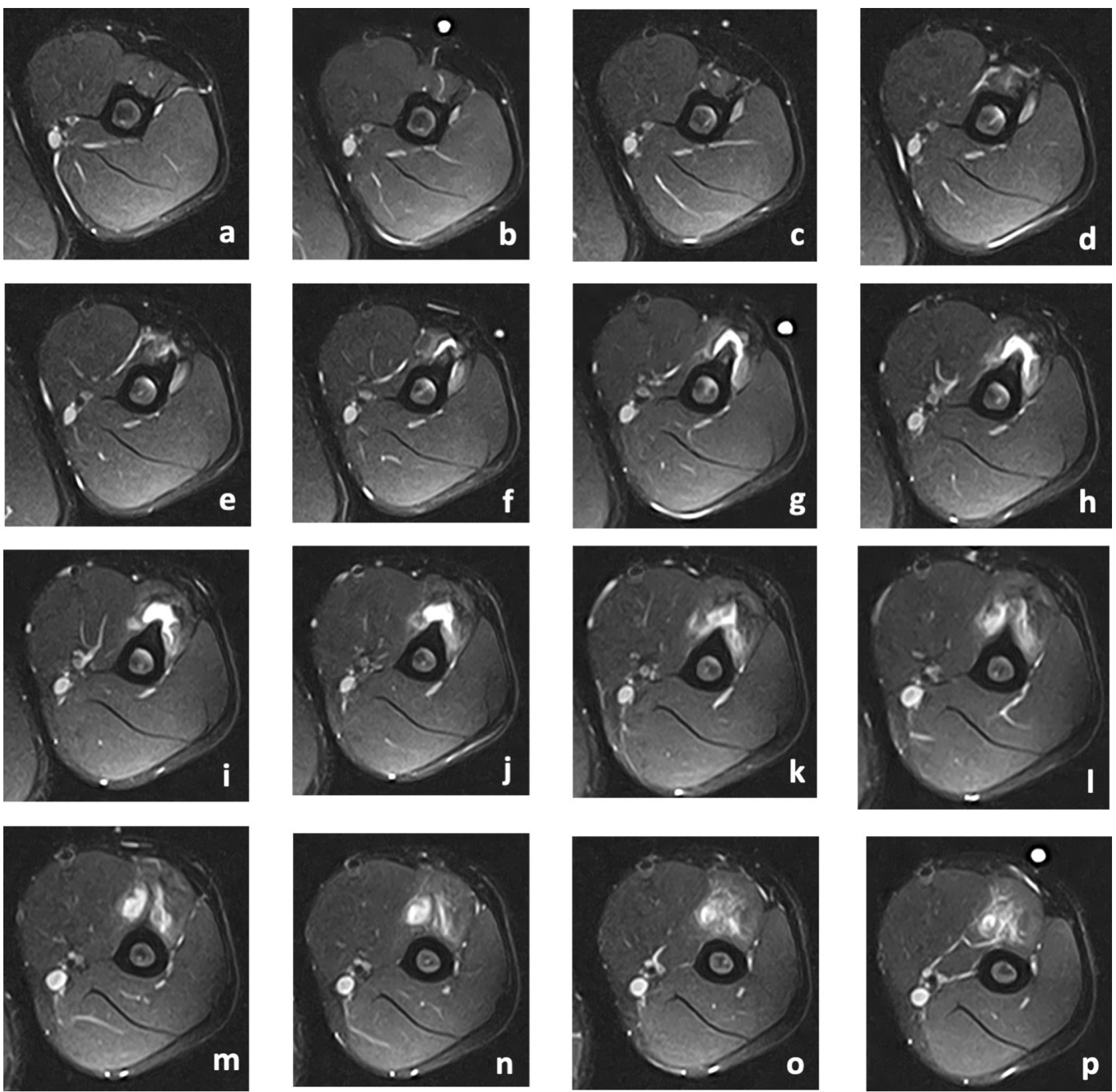

Figure 3: Axial T2 FSAT MRI images (TR: 4,250 ms, TE: $48 \mathrm{~ms}$ ) from most superior (a) to most inferior ( $p$ ), revealing an osseous excrescence in the anterolateral aspect of the humerus near the deltoid attachment. FSAT: Fat-Sat technique.

of stem cells primarily coming from the inner sheet of the periosteum [15]. In the example of a fracture, these progenitor cells proliferate and differentiate, leading to new bone formation at the distant border of the fracture site where the blood supply is adequate and to cartilage formation at the fracture site where the blood supply is poor [16]. If the periosteum remains intact, the subsequent bone formation develops underneath the periosteum [17]. The explanation as to why the ossification of subperiosteal hematomas occurs at the periphery rather than the center is not completely understood, but may be due to the proximity to the inner sheet of periosteum containing the stem cells [18].

The differential diagnosis in this case included myositis ossificans and parosteal osteosarcoma. Myositis ossificans is a self-limited, benign ossifying soft tissue mass that often presents within muscle [19].
Although early stages of myositis ossificans lesions do not demonstrate any ossifications, more mature lesions of six months or greater may demonstrate a densely calcified peripheral rim with a lightly calcified center [19]. Given the origin of the lesion with the muscle, there is often a radiolucent cleft between the ossified mass and the adjacent bone, which may help to differentiate myositis ossificans from a subperiosteal hematoma or a parosteal osteosarcoma [20,21]. Parosteal osteosarcoma is a low-grade, malignant bone tumor that often arises on the metaphyseal surface of long bones [22]. Parosteal osteosarcomas are more commonly found on the distal femoral metadiaphysis followed by the proximal tibial and proximal humeral metadiaphysis [23]. On radiographs, parosteal osteosarcoma often demonstrates a radiodense, lobulated, sessile lesionthat protrudes from the cortex with a broad base attachment [24]. Medullary involvement may also be present. 
Histologically, conventional parosteal osteosarcoma demonstrates irregular bony trabeculae and blandappearing spindle cells within a fibrous stromal tissue [25].

There are multiple features in this case to suggest the diagnosis of an ossified subperiosteal hematoma. First, the patient presented with a chronic history of "calcification" located in the muscle of his left arm that was previously asymptomatic - suggestive of a benign process. Secondly, radiographs demonstrated an thin, well-defined exostosis arising from the cortex of the midhumeral diaphysis. The appearance of the radiodense lesion as well as the mid-diaphyseal location argues against the diagnosis of parosteal osteochondroma. Thirdly, the MRI of the left humerus demonstrated an osseous excrescence in the anterolateral aspect of the humerus and the prominence of the cortex in this area suggested a prior subperiosteal hematoma in this region. The continuation of the lesion with the periosteum argues against the diagnosis of myositis ossificans. The $\mathrm{MRI}$ also demonstrated adventitial bursitis at the tip of the osseous excrescence, which most likely represents the etiology of the patient's pain. Therefore, the authors believe the lesion to be most consistent with an ossified subperiosteal hematoma.

In summary, an ossified subperiosteal hematoma is a benign lesion that commonly presents after direct trauma in young patients without any underlying conditions. This case demonstrates the first reported case of an ossified subperiosteal hematoma of the middiaphyseal region of the humerus bone. A MRI was ordered to confirm the diagnosis and exclude other possible etiologies, including myositis ossificans and parosteal osteochondroma. This patient was treated conservatively, did not demonstrate any complications associated with this lesion, and returned to play lacrosse without any activity restrictions.

\section{References}

1. Chaisrisawadisuk $\mathrm{S}$, Tiong HB, Lipsett $\mathrm{J}$, Anderson PJ (2020) Ossified Encapsulated Cephalhematoma. J Craniofac Surg.

2. Choi J, Lee IW, Yang J, Lee HJ, Yeo IS, et al. (2011) Chronic ossified subperiosteal hematoma of the skull in an 11-yearold child: a case report. Childs Nerv Syst 27: 1165-1168.

3. Currarino G (2007) Neonatal subperiosteal cephalohematoma crossing a synostosed sagittal suture. Pediatr Radiol 37: 1283-1285.

4. Martinez-Lage JF, Esteban JA, Martinez Perez M, Poza M (1984) Craniostenosis secondary to calcified subperiosteal hematoma: case report. Neurosurgery 15: 703-704.

5. Day SB (1960) Ossified subperiosteal hematoma. Observations on three instances including one in an extinct ruminant, the Irish Giant Deer. JAMA 173: 986-990.

6. Herrera-Soto JA, Crawford AH, Loveless EA (2005) Ossifying subperiosteal hematoma associated with neurofibromatosis type 1. Diagnostic hesitation: A case report and literature review. J Pediatr Orthop B 14: 51-54.
7. Hui SH, Lui TH (2016) Subperiosteal Hematoma of the Ankle. J Orthop Case Rep 6: 63-64.

8. Lavell A, Jones CW, Wong D, Counsel P, Carey-Smith $R$ (2017) Plexiform neurofibroma causing an ossifying subperiosteal haematoma: a rare case in the tibia of an 11-year-old girl. Skeletal Radiol 46: 1405-1413.

9. Gürkan V, Ozger H, Berkel T (2007) Ossifying subperiosteal hematoma associated with neurofibromatosis type 1: A report of two cases. Acta Orthop Traumatol Turc 41: 397400.

10. Sakurai A, Masuda T, Yoshiba T, Hanaoka T, Kaneko K (2011) Radiological features of an ossifying subperiosteal hematoma in a patient with severe motor and intellectual disability. J Orthop Sci 16: 334-337.

11. Ben Zakoun J, Dallaudière B, Palazzo E, Lefere $M$, Monteil J, et al. (2014) Chronic ossified subperiosteal hematoma of the iliac bone. Diagn Interv Imaging 95: 889-891.

12. Guillin R, Moser T, Koob M, Khoury V, Chapuis M, et al. (2012) Subperiosteal hematoma of the iliac bone: Imaging features of acute and chronic stages with emphasis on pathophysiology. Skeletal Radiol 41: 667-675.

13. Hughes P, Dow D, Boyer L, Morganti V (2019) Ossifying chronic subperiosteal haematoma of the iliac bone. J Med Imaging Radiat Oncol 63: 479-480.

14. Rodriguez-Merchan EC (2002) Haemophilic cysts (pseudotumours). Haemophilia 8: 393-401.

15. Wang T, Zhang X, Bikle DD (2017) Osteogenic Differentiation of Periosteal Cells During Fracture Healing. J Cell Physiol 232: 913-921.

16. Shapiro $F$ (2008) Bone development and its relation to fracture repair. The role of mesenchymal osteoblasts and surface osteoblasts. Eur Cell Mater 15: 53-76.

17. Smithuis $T$ (1969) Subperiosteal haematoma of the tibia. Report of a case. J Bone Joint Surg Br 51: 130-131.

18. Phillips AM (2005) Overview of the fracture healing cascade. Injury 36 Suppl 3: 5-7.

19. Walczak BE, Johnson CN, Howe BM (2015) Myositis Ossificans. J Am Acad Orthop Surg 23: 612-622.

20. Parikh J, Hyare H, Saifuddin A (2002) The imaging features of post-traumatic myositis ossificans, with emphasis on MRI. Clin Radiol 57: 1058-1066.

21. Thomas EA, Cassar-Pullicino VN, McCall IW (1991) The role of ultrasound in the early diagnosis and management of heterotopic bone formation. Clin Radiol 43: 190-196.

22. Ruengwanichayakun $P$, Gambarotti M, Frisoni T, Gibertoni D, Guaraldi F, et al. (2019) Parosteal osteosarcoma: a monocentric retrospective analysis of 195 patients. Hum Pathol 91: 11-18.

23. Kumar VS, Barwar N, Khan SA (2014) Surface osteosarcomas: Diagnosis, treatment and outcome. Indian J Orthop 48: 255-261.

24. Hang JF, Chen PC (2014) Parosteal osteosarcoma. Arch Pathol Lab Med 138: 694-699.

25. Prabowo Y, Kamal AF, Kodrat E, Prasetyo M, Maruanaya S, et al. (2020)Parosteal Osteosarcoma: A Benign-Looking Tumour, Amenable to a Variety of Surgical Reconstruction. Int J Surg Oncol 2020: 4807612. 